\title{
PROGNOSIS OF PATIENTS RECEIVING INDUCTION CHEMOTHERAPY FOR LOCALLY ADVANCED OR LYMPH-NODE METASTATIC BLADDER CANCER
}

\begin{tabular}{|c|c|}
\hline Journal: & Journal of Clinical Urology \\
\hline Manuscript ID & Draft \\
\hline Manuscript Type: & Cohort Study \\
\hline $\begin{array}{r}\text { Date Submitted by the } \\
\text { Author: }\end{array}$ & $\mathrm{n} / \mathrm{a}$ \\
\hline Complete List of Authors: & $\begin{array}{l}\text { Le Goux, Constance; Hospital Foch, Urology; Versailles Saint-Quentin- } \\
\text { en-Yvelines University Faculty of Medicine Paris Ile de France Ouest, } \\
\text { Urology } \\
\text { Neuzillet, Yann; Hospital Foch, Urology; Versailles Saint-Quentin-en- } \\
\text { Yvelines University Faculty of Medicine Paris Ile de France Ouest, } \\
\text { Urology } \\
\text { Rouanne, Mathieu; Hospital Foch, Urology; Versailles Saint-Quentin-en- } \\
\text { Yvelines University Faculty of Medicine Paris Ile de France Ouest, } \\
\text { Urology } \\
\text { Gachet, Julie; Hospital Foch, Medical Oncology } \\
\text { Staub, Fabrice; Hospital Foch, Radiology } \\
\text { Hervé, Jean-Marie; Hospital Foch, Urology } \\
\text { Yonneau, Laurent; Hospital Foch, Urology } \\
\text { Abdou, Ali; Hospital Foch, Urology } \\
\text { Ghoneim, Tarek; Hospital Foch, Urology } \\
\text { Théodore, Christine; Hospital Foch, Medical Oncology } \\
\text { Lebret, Thierry; Hopital Foch, Urology; Versailles Saint-Quentin-en- } \\
\text { Yvelines University Faculty of Medicine Paris Ile de France Ouest, } \\
\text { Urology }\end{array}$ \\
\hline Keywords: & $\begin{array}{l}\text { muscle invasive bladder cancer, locally advanced bladder cancer, } \\
\text { neoadjuvant chemotherapy, induction chemotherapy, cystectomy }\end{array}$ \\
\hline Abstract: & $\begin{array}{l}\text { Background } \\
\text { Induction chemotherapy is recommended before surgery for } \\
\text { unresectable muscle-invasive bladder cancer (MIBC), locally advanced or } \\
\text { lymph-node disseminated disease. These patient's prognosis cannot be } \\
\text { extrapolated from data regarding neoadjuvant chemotherapy, which is } \\
\text { performed in operable patients. } \\
\text { Objective } \\
\text { We assessed the prognosis of patients undergoing induction } \\
\text { chemotherapy for locally advanced or lymph-node metastatic bladder } \\
\text { cancer. } \\
\text { Methods } \\
\text { We analyzed patients with cT4NxM0 or cTxN+MO bladder cancer treated } \\
\text { by induction chemotherapy between } 2006 \text { and } 2016 \text {. The tumor } \\
\text { extension and node invasion was determined by imaging or histologically } \\
\text { after upfront lymph-node dissection. Clinical, biological, pathological and } \\
\text { patient follow up data were identified. Kaplan-Meier survival curves were } \\
\text { compared by log rank test. Factors associated with the response to } \\
\text { induction chemotherapy, operability of patients and survival were } \\
\text { determined by multivariable logistic regression. }\end{array}$ \\
\hline
\end{tabular}




\section{SCHOLARONE ${ }^{\text {m }}$ Manuscripts}

Results

Among 70 patients included in the analysis, 51 (73\%) showed response to induction chemotherapy. Progression-free and overall survival were improved in responder patients compared to non-responders $(p<0.0001$ and $p=0.025$, respectively) and for patients who underwent surgery compared to the non-operated patients (both $p<0.001$ ). On multivariable analysis, poor response was associated with chemotherapy other than methotrexate, vinblastine, doxorubicin and cisplatin (MVAC) $(p=0.016)$, operability with late response $(p=0.0024)$ and overall survival with surgery after induction chemotherapy $(p=0.0014)$.

Conclusions

Surgery after induction chemotherapy with MVAC may improve prognosis with locally advanced or lymph node metastatic bladder cancer. 
Declaration.

1. None of the authors has a conflict of interest in the topic of this study

2. No funding received

3. No Research involving Human Participants and/or Animals

4. Written informed consent was obtained from the patient(s) for their anonymized information to be published in this article

5. Guarantor : Prof. Thierry LEBRET (TL)

6. Contributorship: CLG Data collection, data analysis and Manuscript writing; YN protocol development, data analysis and project development and Manuscript writing; MR Data collection; JG Data collection; FS Data collection; JMH Data collection; LY Data collection; AA Data collection; TG Data collection and Manuscript Writing; CT Protocol/project development; TL Protocol/project development

7. Acknowledgements: none 


\begin{abstract}
Background

Induction chemotherapy is recommended before surgery for unresectable muscleinvasive bladder cancer (MIBC), locally advanced or lymph-node disseminated disease. These patient's prognosis cannot be extrapolated from data regarding neoadjuvant chemotherapy, which is performed in operable patients.
\end{abstract}

\title{
Objective
}

We assessed the prognosis of patients undergoing induction chemotherapy for locally advanced or lymph-node metastatic bladder cancer.

\section{Methods}

We analyzed patients with cT4NxM0 or $\mathrm{CT} x \mathrm{~N}+\mathrm{M} 0$ bladder cancer treated by induction chemotherapy between 2006 and 2016. The tumor extension and node invasion was determined by imaging or histologically after upfront lymph-node dissection. Clinical, biological, pathological and patient follow up data were identified. Kaplan-Meier survival curves were compared by log rank test. Factors associated with the response to induction chemotherapy, operability of patients and survival were determined by multivariable logistic regression.

\section{Results}


Among 70 patients included in the analysis, 51 (73\%) showed response to induction chemotherapy. Progression-free and overall survival were improved in responder patients compared to non-responders $(p<0.0001$ and $p=0.025$, respectively) and for patients who underwent surgery compared to the non-operated patients (both $\mathrm{p}<0.001$ ). On multivariable analysis, poor response was associated with chemotherapy other than methotrexate, vinblastine, doxorubicin and cisplatin (MVAC) $(p=0.016)$, operability with late response $(p=0.0024)$ and overall survival with surgery after induction chemotherapy $(p=0.0014)$.

\section{Conclusions}

Surgery after induction chemotherapy with MVAC may improve prognosis with locally advanced or lymph node metastatic bladder cancer.

Key words: muscle invasive bladder cancer, locally advanced bladder cancer, neoadjuvant chemotherapy, induction chemotherapy, cystectomy

Level of Evidence: 4 


\section{BACKGROUND}

Unresectable muscle-invasive bladder cancer (MIBC) are defined by a locally advanced disease or associated lymph node metastasis. Unresectable MIBC patients are not considered suitable for upfront surgery and induction chemotherapy is recommended $[1,2]$. The chemotherapy aims to improve the overall survival of the patient, but the potential benefit of the therapy cannot be extrapolated from studies of neoadjuvant chemotherapy, achieved in patients who can immediately undergo surgery. Indeed, whithin the meta-analysis of 2688 patients that reported a 5\% improvement in overall survival with neoadjuvant chemotherapy, only $4 \%$ of patients harbored cN1-3 cancers [3]. Nevertheless, the same cisplatin-based regimens are considered as the gold standard treatment, considering some data showed a comparable objective response rates with gemcitabine-cisplatin and with methotrexate, vinblastine, doxorubicin and cisplatin (MVAC) regimens in induction chemotherapy $[2,4]$. The oncological outcome with chemotherapy regimens without cisplatin have been described as less efficient $[5,6]$. Notwithstanding, some authors recommend the use of regimens without cisplatin, acknowledging that $50 \%$ of patients are unfit for a cisplatin-based chemotherapy in the locally advanced NMIBC setting [4, 7-9]. The rational for performing such induction chemotherapy without cisplatin, is that suboptimal chemotherapy is better than nothing.

Irrespective of the kind of chemotherapy regimen, the optimal number of cycles for induction chemotherapy has not been determined. That number must reconcile two objectives: in one hand, an objective response must be observed, meaning that the 
amount of cycle is sufficient; in the other hand, the patient's overall health must be consistent with the radical surgery. In current practices, induction chemotherapy involves 4 to 8 cycles. No evidence exists regarding the optimal protocol for this induction chemotherapy and surgery combination, and the subsequent outcome at the end of this treatment.

Bearing in mind the need investigates the objective response and prognosis of patients receiving induction chemotherapy for MIBC non-eligible for upfront surgery, the objectives of this study were to define the objective response rate during induction chemotherapy, and the subsequent progression-free survival (PFS) and overall survival (OS) according both to the response to induction chemotherapy, and the completion of the radical cystectomy.

\section{MATERIALS AND METHODS}

\section{$\underline{\text { Patients and Samples }}$}

Data from patients with locally advanced (cT4NxM0) or lymph-node invasion (cTxN+) MIBC treated with induction chemotherapy in our center between September 2006 and February 2016 were collected. The study was approved by the local ethic and research committee. Tumors were graded according to the OMS 2004 tumor-grading scheme [10] and were re-staged according to the 2009 TNM classification of bladder tumors [11]. The tumor and lymph-node invasion were determined by either CT or PET/CT. 
Alternatively, lymph-node invasion was determined histologically with a upfront lymph-node dissection.

The objective response to induction chemotherapy was assessed through repeated CT or PET /CT (according to modality used for initial staging), and performed between the third and the sixth cycles of induction chemotherapy. For conventional imaging, the response to induction chemotherapy was defined as complete response or partial response according to RECIST 1.1 criteria [12]. Using PET/CT, complete response was defined by the total disappearance of abnormal uptake $[13,14]$. Surgery could be performed when objective response allowed the resection of all lesions defined on imaging. Late response was defined surgery was allowed when achieved after more than 8 courses of induction chemotherapy. Standard follow-up visits were performed according to current guidelines.

The studied variables were:

- Cinical features:

- Number of patients

- Age

- Sex

- Smoking status

- Professional exposure

- History of bladder tumor

- Body Mass Index (BMI) 
- Performance status (PS) according to the WHO classification

- Biological features:

- Creatininemia with Cockcroft and Gault creatinine clearance calculation (normal renal function when clearance was $\geq 90 \mathrm{~mL} / \mathrm{min}$, renal insufficiency stage I when $60 \leq$ clearance $\leq 89 \mathrm{~mL} / \mathrm{min}$, stage II when $30 \leq$ clearance $\leq 59 \mathrm{~mL} / \mathrm{min}$, stage III when clearance $<30 \mathrm{~mL} / \mathrm{min}$ )

- Nutritional status (denutrition when albuminemia $<40 \mathrm{~g} / \mathrm{L}$ )

- Blood counts (anemia when hemoglobin was $<12 \mathrm{~g} / \mathrm{dL}$, normal platelet count between 150 and $400 \mathrm{G} / \mathrm{L}$, normal leukocyte count between 4 and $10 \mathrm{G} / \mathrm{L}$ )

- Induction chemotherapy features:

- Type of chemotherapy

- Timing of re-evaluation by imagery

- Number of cycles

- Pathologic and operating features:

- Lymph-node dissection

- Pathologic data pre and post-cystectomy (in case of cystectomy):

- Histological type

- TNM

- Margins after cystectomy

- Prostate cancer seen on specimens

- Follow-up

- The occurrence of recurrence specifying the site 
- Date of latest news

- The date of death and its cause (related or unrelated to urothelial disease)

\section{$\underline{\text { Statistical analyses }}$}

Data are reported with mean (range). The clinicopathologic features of MIBC were tested for their association with induction chemotherapy response and surgery completion by chi-square test. PFS was defined as the time from the date of chemotherapy response to the first local relapse or first metastasis. OS was calculated from the date of diagnosis to death or the last follow-up. Patients were censored if they had not experienced the end-point of interest at the time of the last follow-up. Kaplan-Meier survival curves were compared by log rank test. The impact of clinicopathologic features on induction chemotherapy response, cystectomy and survival was assessed by multivariable analysis with a Cox proportional-hazards regression model, estimating hazard ratios (HRs). Factors significant on univariate analysis at $p<0.10$ were included in the multivariable analysis. $p<0.05$ was considered statistically significant.

\section{RESULTS}




\section{Patients}

In total, we identified 72 patients with locally advanced bladder tumour and/or $\mathrm{N}+$ status who should receive induction chemotherapy over the study period; 70 (mean age 65 years [median 66 years (33-86)]) actually received induction chemotherapy (Figure 1). The mean follow-up was 31 months (median 27 months [5-108]).

\section{Induction chemotherapy protocols and response rates}

Induction chemotherapy consisted in cisplatin-based protocols for 52 patients (50 MVAC, 1 Cisplatine-Etoposid, 1 Cisplatine-Laroxel) (74.3\%) and taxol-gemcitabine protocol for 18 patients (25.7\%). Overall, an objective response to induction chemotherapy were observed in 51 patients (72.9\%). The clinical, biologic, and pathologic features of responders and non-responders are reported in Table 1 . The mean number of chemotherapy cycles received among responders was 7 (median 5 [4-29]) comparing to 21 (median 16 [4-70]) among non-responders. Clinical factors significantly associated with response to induction chemotherapy were sex, smoking status and induction chemotherapy using MVAC regimen. The only biological factor significantly associated with response to chemotherapy was kidney function. On multivariable analysis, response to induction chemotherapy was associated with MVAC as first-line chemotherapy (Table 2a).

\section{Surgery completion}

Forty-seven patients underwent surgery (44/51 responders [86\%] and 3/19 nonresponders [16\%]). The clinical, biologic and pathologic features of the 70 patients 
according to surgery completion are reported in Table 3. Among the 3 non-responders, 2 underwent surgery due to an uncontrollable hematuria requiring iterative transfusions, and the last was operated after an initial unresponsiveness under induction therapy with laroxel-cisplatin then a response under a second-line therapy with taxol-gemcitabine. Overall, 45/47 patients underwent lymph-node dissection; The 2 patient who did not have lymph-node dissection were those requiring cystectomy for bleeding control. There were no deaths in early post-operative period ( $<30$ days). Factors associated with surgery completion after induction chemotherapy were age, sex, smoking status, response to chemotherapy, regardless of the time of the response, and histologic urothelial type. On multivariable analysis, surgery completion inversely associated with late response to induction chemotherapy (Table 2b).

\section{Outcome according to response to induction chemotherapy and surgery}

\section{completion}

Regardless surgery completion, patients with response to induction chemotherapy showed improved PFS ( $p<0.001$; Figure 2a) and OS ( $p=0.025$; Figure $2 b)$. regardless response to induction chemotherapy, patients who underwent surgery showed improved PFS $(p<0.001)$ and OS $(p<0.001)$. Professional exposure was the only prognostic factor significantly associated with lower PFS (Table 4a).. Surgery completion after induction chemotherapy was the only prognostic factor significantly associated with OS (Table 4b). Surgery after induction chemotherapy was protective for risk of death. 


\section{DISCUSSION}

Concept of unresectable MIBC patients includes both locally advanced diseases, which invade pelvic organs, and lymph nodes invasive diseases, where extent of lymph nodes dissection that should be done to achieve a complete resection of the cancer is too morbid and with uncertain efficacy. The possibility to achieve a complete surgical resection of the disease depends both of the ability of the surgical team in performing complex procedure, and the physical capacity and psychological acceptance of the patients regarding the surgical treatment. Each of these criteria may vary widely according to the medico-economic and sociologic environments. Thus, limited epidemiological data on a such heterogeneous disease are available, and therapeutics guidelines in this setting are primarily based on expert advises. Data from Surveillance, Epidemiology and End Results database reported by Abdollah et al. had estimated prevalence and incidence of regional MIBC at 26,014 cases and 4.7 new cases/years/100 000 US citizens [15]. Studies focusing on induction chemotherapy for locally advanced or lymph-node metastatic MIBC are relatively old and have included limited in number of patients, with methodological issues [16-23]. Overall, the objective response rate to induction chemotherapy ranges from $33 \%$ within the historical cohort described by Herr et al. [23], to $44 \%-86 \%$ in most recent series [16-22]. In accordance with these previous studies, we report an objective response rate of $72.9 \%$. In most of the previous studies, objective response to induction chemotherapy was associated with improved survival. The results from the present study confirm improvement in 
overall survival in patients who obtain an objective response through induction chemotherapy. Moreover, our results showed an independent association between the response to induction chemotherapy and use of MVAC regimen contrary to others regimens. Our study also demonstrates an improvement in overall survival in patients who underwent surgery after induction chemotherapy. In addition, we showed that late response to induction chemotherapy was independently associated with reduced probability for surgery completion. Taken together, these results defend a maximalist approach, combining chemotherapy and surgery in the treatment of unresectable MIBC.

Use of induction chemotherapy is based on pragmatic principle that as the extent of cancer is too important for a surgical resection, hence an upfront chemical reduction of this extend is aimed [24]. Cisplatin based chemotherapy regimens have demonstrated improvement of overall survival in patients with metastatic diseases [2]. Contrary to chemotherapy, evidences of clinical benefit of surgical resection of tumors in this setting did not concern overall survival improvement, but control of symptoms, mainly bleeding. Indeed, in the locally unresectable MIBC setting (i.e. cT4b tumors), extirpative surgery alone, not combined with chemotherapy, has demonstrated futility in many studies [25-27]. Pak et al. recently reports results from a retrospective study comparing survival outcomes in patients with clinically node-positive muscle-invasive bladder cancer receiving induction chemotherapy followed by surgery and those who underwent upfront radical cystectomy. Through the analyses of 340 patients, including 
106 received induction chemotherapy and 234 underwent upfront surgery, authors shown that the 5-year cancer-specific survival rate of cN1-2 patients was higher in the induction chemotherapy group than the upfront surgery group (68.1\% vs. 52.9\%). Our results reinforce the conclusion of previous studies, in supporting combination of both chemotherapy then surgery. Indeed, through a systematic review of literature, cumulating 7 retrospective studies, Li et al. reported that this strategy may improve survival in patient with $\mathrm{cT} \leq 3 \mathrm{bN}+\mathrm{MO} \mathrm{MIBC}$ [28]. The results from the present study bring new contribution in patients management regarding timing for surgery. Indeed, our multivariable analysis shown that surgery completion was inversely associated with late response to induction chemotherapy, meaning that in prolonging the chemotherapy, probability of completing surgery completion is not improved. Thus, objective response should be early assessed in order to perform surgery as soon as tumor removability is obtained.

This study had several limitations. Mainly, this study was retrospective in nature and may therefore be subject to inherent bias. Inherent limitations may have included selection bias for induction or adjuvant chemotherapy and the lack of prospective standardized protocols for lymphadenectomy, chemotherapy regimen, and salvage treatments. 


\section{CONCLUSIONS}

Patients with unresectable MIBC constitute a problematic group of patients which require a multimodal treatment. If cisplatin-based induction chemotherapy had demonstrated a keen interest regarding overall survival, our study adds argument in favor of benefit of subsequent surgery. This surgery should not be delayed in prolonging induction chemotherapy beyond a sufficient clinical response, allowing to remove the tumors. The extirpability status of tumors could be dependant of the surgical team, thus patients applicant to this kind of surgery could benefit by being performed in tertiary center. 


\section{REFERENCES}

1. Bamias A, Tiliakos I, Karali M-D, Dimopoulos MA. Systemic chemotherapy in inoperable or metastatic bladder cancer. Ann Oncol Off J Eur Soc Med Oncol. 2006;17:553-61.

2. von der Maase H, Sengelov L, Roberts JT, Ricci S, Dogliotti L, Oliver T, et al. Longterm survival results of a randomized trial comparing gemcitabine plus cisplatin, with methotrexate, vinblastine, doxorubicin, plus cisplatin in patients with bladder cancer. J Clin Oncol Off J Am Soc Clin Oncol. 2005;23:4602-8.

3. Advanced Bladder Cancer (ABC) Meta-analysis Collaboration. Neoadjuvant chemotherapy in invasive bladder cancer: update of a systematic review and meta-analysis of individual patient data advanced bladder cancer ( $A B C)$ metaanalysis collaboration. Eur Urol. 2005;48:202-5; discussion 205-206.

4. Zargar H, Espiritu PN, Fairey AS, Mertens LS, Dinney $C P$, Mir MC, et al. Multicenter assessment of neoadjuvant chemotherapy for muscle-invasive bladder cancer. Eur Urol. 2015;67:241-9.

5. Linardou $H$, Aravantinos G, Efstathiou E, Kalofonos C, Anagnostopoulos A, Deliveliotis $C$, et al. Gemcitabine and carboplatin combination as first-line treatment in elderly patients and those unfit for cisplatin-based chemotherapy with advanced bladder carcinoma: Phase II study of the Hellenic Co-operative Oncology Group. Urology. 2004;64:479-84.

6. Dogliotti L, Cartenì G, Siena S, Bertetto O, Martoni A, Bono A, et al. Gemcitabine 
plus cisplatin versus gemcitabine plus carboplatin as first-line chemotherapy in advanced transitional cell carcinoma of the urothelium: results of a randomized phase 2 trial. Eur Urol. 2007;52:134-41.

7. Apolo AB, Grossman HB, Bajorin D, Steinberg G, Kamat AM. Practical use of perioperative chemotherapy for muscle-invasive bladder cancer: summary of session at the Society of Urologic Oncology annual meeting. Urol Oncol. 2012;30:772-80.

8. See WA. Commentary on "Carboplatin based induction chemotherapy for nonorgan confined bladder cancer--a reasonable alternative for cisplatin unfit patients?" Mertens LS, Meijer RP, Kerst JM, Bergman AM, van Tinteren $H$, van Rhijn BW, Horenblas S, Department of Urology, The Netherlands Cancer Institute, Antoni van Leeuwenhoek Hospital, Amsterdam, The Netherlands: J Urol 2012;188(4):1108-13 [Epub 2012 Aug 15]. Urol Oncol. 2013;31:716-7.

9. Mertens LS, Meijer RP, Kerst JM, Bergman AM, van Tinteren $H$, van Rhijn BWG, et al. Carboplatin based induction chemotherapy for nonorgan confined bladder cancer--a reasonable alternative for cisplatin unfit patients? J Urol. 2012;188:1108-13.

10. Molinié V. Classification of bladder tumors in 2006. In: Prog Urol FMC. 2006. p. 16:7-10.

11. Cheng L, Montironi R, Davidson DD, Lopez-Beltran A. Staging and reporting of urothelial carcinoma of the urinary bladder. Mod Pathol Off J U S Can Acad Pathol Inc. 2009;22 Suppl 2:S70-95. 
12. Eisenhauer EA, Therasse P, Bogaerts J, Schwartz LH, Sargent D, Ford R, et al. New response evaluation criteria in solid tumours: revised RECIST guideline (version 1.1). Eur J Cancer Oxf Engl 1990. 2009;45:228-47.

13. Kollberg P, Almquist H, Bläckberg M, Cwikiel M, Gudjonsson S, Lyttkens K, et al. [18F]Fluorodeoxyglucose-positron emission tomography/computed tomography response evaluation can predict histological response at surgery after induction chemotherapy for oligometastatic bladder cancer. Scand J Urol. 2017;51:308-13.

14. Mertens LS, Fioole-Bruining A, Vegt E, Vogel WV, van Rhijn BW, Horenblas S. Impact of (18) F-fluorodeoxyglucose (FDG)-positron-emission tomography/computed tomography (PET/CT) on management of patients with carcinoma invading bladder muscle. BJU Int. 2013;112:729-34.

15. Abdollah F1, Gandaglia G, Thuret R, Schmitges J, Tian Z, Jeldres C, et al. Incidence, survival and mortality rates of stage-specific bladder cancer in United States: a trend analysis. Cancer Epidemiol. 2013;37:219-25.

16. Galsky MD, Stensland K, Sfakianos JP, et al. Comparative effectiveness of treatment strategies for bladder cancer with clinical evidence of regional lymph node involvement. Journal of Clinical Oncology. 2016;34:2627.

17. Zargar-Shoshtari $\mathrm{K}$, Zargar $\mathrm{H}$, Lotan $\mathrm{Y}$, et al. A multi-institutional analysis of outcomes of patients with clinically node positive urothelial bladder cancer treated with induction chemotherapy and radical cystectomy. The Journal of urology. 2016;195:53-59. 
18. Ho PL, Willis DL, Patil J, et al. Outcome of patients with clinically node-positive bladder cancer undergoing consolidative surgery after preoperative chemotherapy: The MD Anderson Cancer Center Experience. Urologic Oncology: Seminars and Original Investigations. Vol 34: Elsevier; 2016:59. e5159. e58.

19. Hermans TJ, van de Putte EEF, Horenblas S, et al. Pathological downstaging and survival after induction chemotherapy and radical cystectomy for clinically node-positive bladder cancerDResults of a nationwide population-based study. European Journal of Cancer. 2016;69:1-8.

20. Urakami S, Yuasa T, Yamamoto S, et al. Clinical response to induction chemotherapy predicts improved survival outcome in urothelial carcinoma with clinical lymph nodal metastasis treated by consolidative surgery. International journal of clinical oncology. 2015;20:1171-1178.

21. Meijer RP, Mertens LS, van Rhijn BW, et al. Induction chemotherapy followed by surgery in node positive bladder cancer. Urology. 2014;83:134-139.

22. Nieuwenhuijzen J, Bex A, Meinhardt W, et al. Neoadjuvant methotrexate, vinblastine, doxorubicin and cisplatin for histologically proven lymph node positive bladder cancer. The Journal of urology. 2005;174:80-84

23. Herr HW, Donat SM, Bajorin DF. Post-chemotherapy surgery in patients with unresectable or regionally metastatic bladder cancer. The Journal of urology. 2001;165:811-814

24. Fosså SD, Sternberg C, Scher HI, Theodore CH, Mead B, Dearnaley D, Roberts JT, 
Skovlund E. Survival of patients with advanced urothelial cancer treated with cisplatin-based chemotherapy. Br J Cancer. 1996;74:1655-9.

25. Furukawa J, Miyake H, Hara I, Takenaka A, Fujisawa M. Clinical outcome of radical cystectomy for patients with pT4 bladder cancer. Int J Urol. 2008;15:58-61.

26. Liberman D, Alasker A, Sun M, Ismail S, Lughezzani G, Jeldres C, Budaus L, Thuret R, Shariat SF, Widmer H, Perrotte P, Graefen M, Montorsi F, Karakiewicz PI. Radical cystectomy for patients with pT4 urothelial carcinoma in a large population-based study. BJU Int. 2011;107:905-11.

27. Tilki D, Svatek RS, Karakiewicz PI, Isbarn H, Reich O, Kassouf W, Fradet $Y$, Novara G, Fritsche HM, Bastian PJ, Izawa JI, Stief CG, Ficarra V, Lerner SP, Schoenberg M, Dinney CP, Skinner E, Lotan Y, Sagalowsky AI, Shariat SF. Characteristics and outcomes of patients with pT4 urothelial carcinoma at radical cystectomy: a retrospective international study of 583 patients. J Urol. 2010;183:87-93.

28. Li R, Metcalfe M, Kukreja J, Navai N. Role of Radical Cystectomy in Non-Organ Confined Bladder Cancer: A Systematic Review. Bladder Cancer. 2018;4:31-40. 


\section{ABBREVIATIONS}

BMI: Body Mass Index

$\mathrm{CT}$ : computer tomography

(HD)-MVAC: high-dose methotrexate, vinblastine, doxorubicin and cisplatin

EORTC: European Organisation for Research and Treatment of Cancer

INF $\alpha$ : interferon $\alpha$

MIBC: muscle-invasive bladder cancer

MRI: Magnetic Resonance Imaging

MVAC: methotrexate, vinblastine, doxorubicin and cisplatin

NMIBC: non-muscle-invasive bladder cancer

OMS: Organisation Mondiale de la Santé

OS: overall survival

PET: positron emission tomography

PFS: progression-free survival

PS: Performans status

RECIST: response evaluation criteria in solid tumours

SEER: The Surveillance, Epidemiology, and End Results

SWOG: Southwest Oncology Group

TNM: Tumor Node Metastasis 
Table 1: Clinical, biologic and pathologic features of 70 patients with and without response to induction chemotherapy

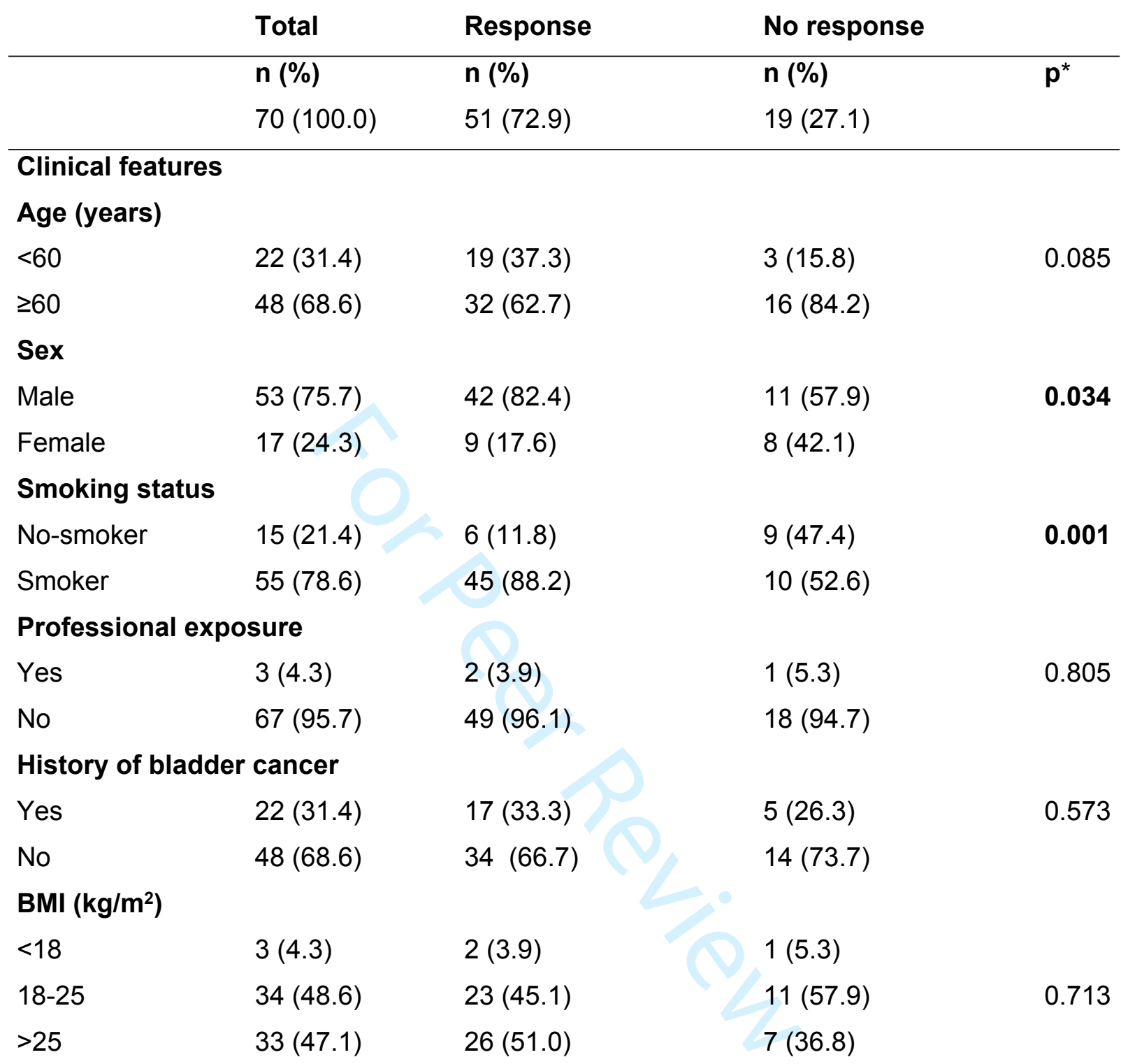

\section{Performance status}

$\begin{array}{lllll}0-1 & 64(91.4) & 49(96.1) & 15(78.9) & 0.372 \\ 2-3 & 6(8.6) & 2(3.9) & 4(21.1) & \end{array}$

Type of first-line chemotherapy

$\begin{array}{llll}\text { MVAC } & 50(71.4) & 42(82.4) & 8(42.1) \\ \text { TG } & 18(25.7) & 9(17.6) & 9(47.4) \\ \text { Other } & 2(2.9) & 0(0.0) & 2(10.5)\end{array}$

Biologic features

Renal failure

Yes

No

$13(18.6)$

13 (25.5)

$0(0.0)$ 


\section{Denutrition ${ }^{£}$}

Yes 27 (90.3)

17 (81.0)

$10(100.0)$

0.139

No

$$
4 \text { (9.7) }
$$

4 (19.0)

$0(0.0)$

Anemia ${ }^{£}$

Yes

27 (40.3)

18 (37.5)

9 (47.4)

0.458

No

40 (59.7)

30 (62.5)

$10(52.6)$

Platelets $£ £ £$

Thrombocytopenia 3 (4.6)

$2(4.3)$

1 (5.6)

Normal rate

53 (81.5)

$38(80.8)$

15 (83.3)

0.908

Thrombocytosis

9 (13.8)

7 (14.9)

2 (11.1)

Leukocytes ${ }^{£ £ £ £ ~}$

Leukopenia

$$
2 \text { (3.3) }
$$

Normal rate

45 (73.8)

$32(71.1)$

$13(81.3)$

0.346

Leukocytosis

$14(22.9)$

$12(26.7)$

2 (12.5)

Anatomo-pathological features

Histologic type

Urothelial

66 (94.3)

49 (96.1)

17 (89.5)

Other

4 (5.7)

2 (3.9)

2 (10.5)

0.290

Tumour stage

(cT)

$\begin{array}{llll}<\text { T3 } & 46(65.7) & 36(70.6) & 10(52.6) \\ \text { T3 } & 9(12.9) & 7(13.7) & 2(10.5) \\ \text { T4 } & 15(21.4) & 8(15.7) & 7(36.8)\end{array}$

0.159

Lymph node status

$\begin{array}{lllll}\mathrm{N}- & 6(8.6) & 3(5.9) & 3(15.8) & 0.188 \\ \mathrm{~N}+ & 64(91.4) & 48(94.1) & 16(84.2) & \end{array}$

MVAC, methotrexate, vinblastine, doxorubicin and cisplatin; TG, taxol-gemcitabine

* $\mathrm{Chi}^{2}$ test

${ }^{£}$ data available for 31 patients

££ data available for 67 patients

£££ data available for 65 patients

ffef data available for 61 patients

ffeff data available for 41 patients 
Table 2: a) Multivariable analysis of factors associated with response to induction chemotherapy

\begin{tabular}{lll}
\hline Pronostic factor & \multicolumn{2}{l}{ Response to chemotherapy } \\
& $H R$ & $\mathrm{p}$ \\
\hline Age & 2.98 & 0.22 \\
Sex & 1.90 & 0.44 \\
Smoking status & 3.25 & 0.16 \\
Type of first-line chemotherapy & & $\mathbf{0 . 0 1 6}$ \\
MVAC & 1 & \\
TG & 0.05 & \\
Other & 0.01 & \\
Renal function & 0.00 & 0.98
\end{tabular}

MVAC, methotrexate, vinblastine, doxorubicin and cisplatin; TG, taxol-gemcitabine $\mathrm{HR}$, hazard ratio; $95 \% \mathrm{Cl}, 95 \%$ confidence interval

Table 2: b) Multivariable analysis of factors associated with surgery completion.

\begin{tabular}{lll}
\hline Pronostic factor & Operability & \\
& $H R$ & $\mathrm{p}$ \\
\hline Age & 125.90 & 0.98 \\
Sex & 0.98 & 0.96 \\
Smoking status & 0.40 & 0.59 \\
BMI (kg/m $\left.{ }^{2}\right)$ & & 0.76 \\
$<18$ & 1.00 & \\
$18-25$ & 0.74 & \\
$>25$ & 0.41 & \\
Performance status before chemotherapy & 0.12 & 0.23 \\
Performance status after chemotherapy & 0.00 & 0.98 \\
Response to chemotherapy & 3.00 & 0.44 \\
Late response to chemotherapy & 0.01 & $\mathbf{0 . 0 2 4}$ \\
Histologic type & 1.02 & 0.99 \\
\hline
\end{tabular}


Table 3: Clinical, biologic and pathologic characteristics of 70 patients after induction therapy with and without cystectomy

\begin{tabular}{lllll}
\hline & General & Surgery & No Surgery & \\
\hline $\mathbf{n}(\%)$ & $\mathbf{n}(\%)$ & $\mathbf{n}(\%)$ & $\mathbf{p}^{*}$ \\
\hline $70(100.0)$ & $47(67.1)$ & $23(32.9)$ & \\
\end{tabular}

Clinical features

Age (years)

$<60$

$20(28.6) \quad 17(36.2)$

$3(13.0)$

0.044

$\geq 60$

$50(71.4) \quad 30(63.8)$

$20(87.0)$

Sex

Male

$53(75.7) \quad 40(85.1)$

13 (56.5)

0.009

Female

17 (24.3)

7 (14.9)

10 (43.5)

\section{Smoking status}

No-smoker

$15(21.4)$

$6(12.8)$

9 (39.1)

0.001

Smoker

55 (78.6)

41 (87.2)

14 (60.9)

Professional exposure

Yes

3 (4.3)

2 (4.3)

$1(4.4)$

0.986

No

67 (95.7)

45 (95.7)

22 (95.6)

\section{History of bladder}

cancer

Yes

$22(31.4) \quad 16(34.0)$

6 (26.1)

0.500

No

48 (68.6)

31 (66.0)

$17(73.9)$

BMI $\left(\mathrm{kg} / \mathrm{m}^{2}\right)$

$<18$

3 (4.3)

2 (4.2)

1 (4.3)

18-25

34 (48.6)

20 (42.6)

$14(60.9)$

0.086

$>25$

33 (47.1)

25 (53.2)

8 (34.8)

Performance status

0-1

$64(91.4) \quad 45(95.7)$

19 (82.6)

0.065

2-3

6 (8.6)

$2(4.3)$

4 (17.4)

Type of $1^{\text {st }}$ line chemotherapy

$\begin{array}{lllll}\text { MVAC } & 50(71.4) & 37(78.7) & 13(56.5) & \\ \text { TG } & 18(25.7) & 9(19.1) & 9(39.1) & 0.155 \\ \text { Other } & 2(2.9) & 1(2.1) & 1(43.5) & \end{array}$

Response to induction chemotherapy

Yes

$51(72.9)$

$44(93.6)$

7 (30.4)

$<0.001$ 
No

$$
19(27.1)
$$

$3(6.4)$

$16(69.6)$

Late response to chemotherapy $₹$

Yes

7 (13.7)

2 (4.5)

5 (71.4)

$<0.001$

No

44 (86.3)

42 (95.5)

2 (28.6)

BMI post-chemotherapy $\left(\mathrm{kg} / \mathrm{m}^{2}\right)$

$\begin{array}{lllll}<18 & 1(1.4) & 1(2.1) & 0(0.0) & \\ 18-25 & 42(60.0) & 28(59.6) & 14(60.9) & 0.780 \\ >25 & 27(38.6) & 18(38.3) & 9(39.1) & \end{array}$

Performance status post-

chemotherapy

0-1

$65(92.9) \quad 46(97.9)$

19 (82.6)

0.020

2-3

5 (7.1)

$1(2.1)$

4 (17.4)

Biologic features

Renal failure

Yes

57 (81.4)

36 (76.6)

21 (91.3)

0.137

No

13 (18.6)

$11(23.4)$

2 (8.7)

Denutrition ${ }^{\mathfrak{}}$

Yes

27 (90.3)

17 (81.0)

$10(100.0)$

0.139

No

$4(9.7)$

$4(19.0)$

$0(0.0)$

Anemia ${ }^{£ £}$

Yes

28 (41.8)

$18(40.0)$

$10(45.5)$

0.670

No

39 (58.2)

27 (60.0)

$12(54.5)$

Platelets ${ }^{£ £ £ £ ~}$

Thrombocytoopenia

3 (4.6)

$2(4.5)$

$1(4.8)$

Normal rate

$53(81.5)$

37 (84.1)

$16(76.2)$

0.699

Thrombocytosis

9 (13.8)

$5(11.4)$

$4(19.0)$

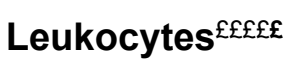

Leukopenia

$$
2(3.3)
$$

2 (4.3)

$0(0.0)$

Normal rate

45 (73.8)

$29(61.7)$

$16(84.2)$

0.381

Leukocytosis

$14(22.9)$

$11(23.4)$

$3(15.8)$

Anatomic-pathological features

Histologic type

Urothelial

Other

66 (94.3)

4 (5.7)

$46(65.7)$

$$
<\text { T3 }
$$

Tumour stage (cT)

19 (82.6)

$0(0.0)$

$4(17.4)$

0.003 
2

T4

\section{Lymph node status}

$\begin{array}{llll}\mathrm{N}- & 6(8.6) & 4(8.5) & 2(8.7) \\ \mathrm{N}+ & 64(91.4) & 43(91.5) & 21(91.3)\end{array}$

\footnotetext{
* Chi $^{2}$ test

${ }^{£}$ Among 51 patients responders to induction chemotherapy

${ }^{£ £}$ Information available for 31 patients

${ }^{£ £ \in ~ I n f o r m a t i o n ~ a v a i l a b l e ~ f o r ~} 67$ patients

£fff Information available for 65 patients

£ffef Information available for 61 patients

£££ff£ Information available for 41 patients
}

MVAC, methotrexate, vinblastine, doxorubicin and cisplatin; TG, taxol-gemcitabine 
Table 4: a) Multivariable analysis of factors associated with progression-free survival

\begin{tabular}{lll}
\hline Pronostic factor & \multicolumn{2}{l}{ Progression-free survival } \\
& $H R$ & $\mathrm{p}$ \\
\hline Smoking status & 0.60 & 0.23 \\
Professional exposure & 7.29 & $\mathbf{0 . 0 0 3 8}$ \\
Histologic type & 1.19 & 0.84 \\
Tumour stage (cT) & & 0.21 \\
$<$ T3 & 1 & \\
T3 & 1.29 & \\
T4 & 1.67 & \\
Platelets & & 0.093 \\
Thrombocytopenia & 1 & \\
Normal rate & 0.42 & \\
Thrombocytosis & 0.18 & \\
Response to chemotherapy & 2.13 & 0.13 \\
Late response to chemotherapy & 1.13 & 0.081 \\
Cystectomy after chemotherapy & 2.31 & 0.086 \\
& &
\end{tabular}

Table 4: b) Multivariable analysis of factors associated with overall survival

\begin{tabular}{llc}
\hline Pronostic factor & \multicolumn{2}{c}{ Overall survival } \\
& $H R$ & $\mathrm{p}$ \\
\hline Sex & 0.47 & 0.23 \\
Platelets & & 0.50 \\
Thrombocytopenia & 1 & \\
Normal rate & 0.56 & \\
Thrombocytosis & 0.31 & \\
Response to chemotherapy & 0.45 & 0.35 \\
Late response to chemotherapy & 0.30 & 0.22 \\
Cystectomy after chemotherapy & 0.21 & $\mathbf{0 . 0 1 1}$
\end{tabular}




\section{Figure 1: Study flow chart}

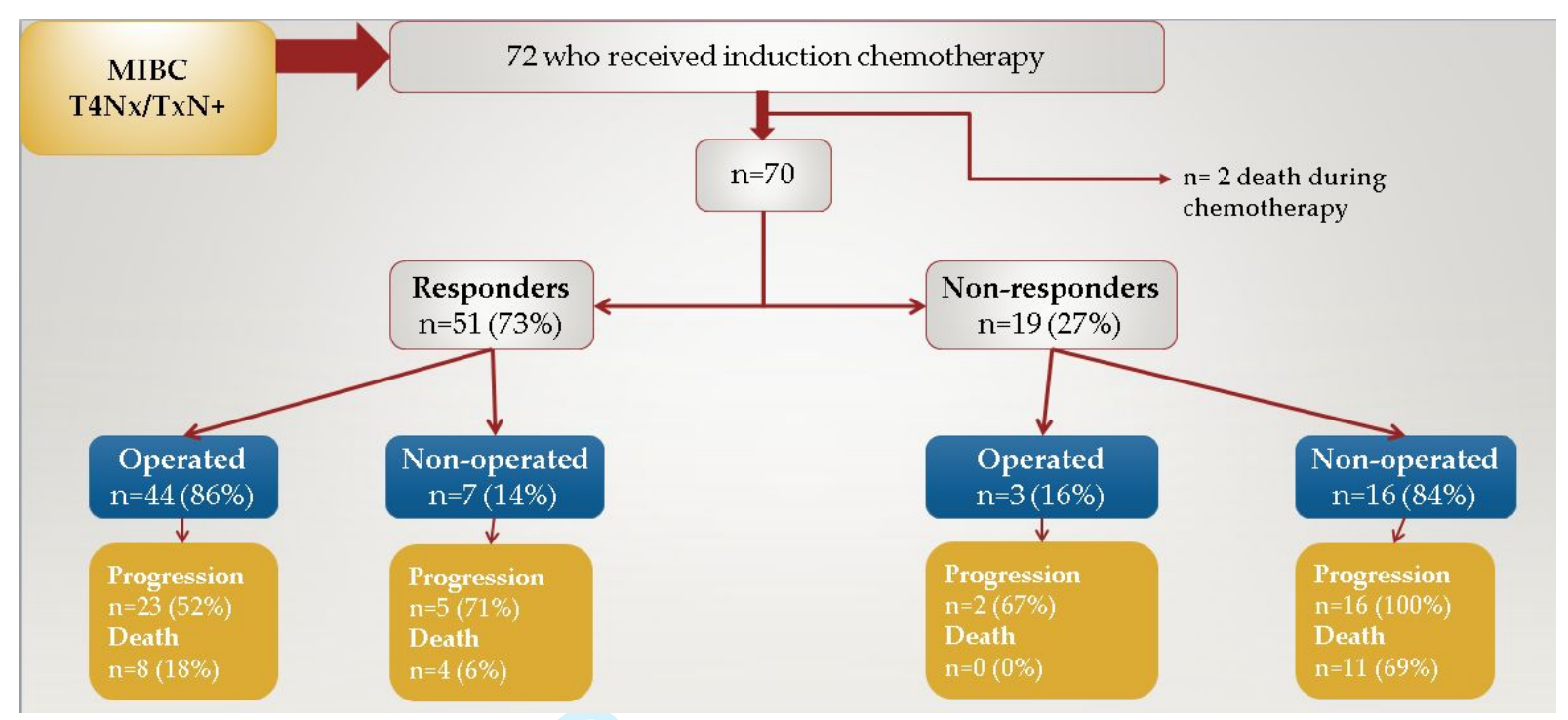


Figure 2a: Progression-free survival according to the response to induction chemotherapy

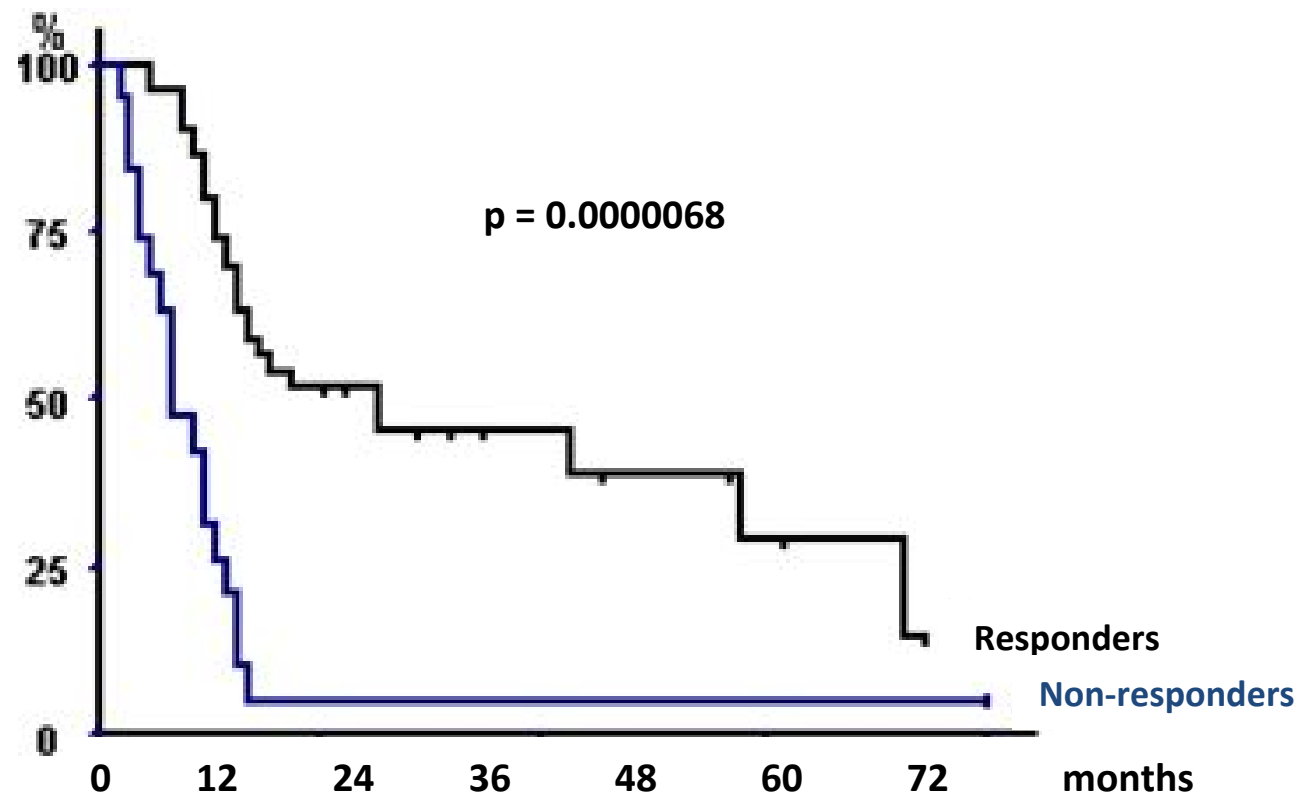


Page 31 of 32

Journal of Clinical Urology

$$
\begin{aligned}
& 1 \\
& 2 \\
& 3 \\
& 4 \\
& 5 \\
& 6 \\
& 7 \\
& 8 \\
& 9 \\
& 10 \\
& 11 \\
& 12 \\
& 13 \\
& 14 \\
& 15 \\
& 16 \\
& 17 \\
& 18 \\
& 19 \\
& 20 \\
& 21 \\
& 22 \\
& 23 \\
& 24 \\
& 25 \\
& 26 \\
& 27 \\
& 28 \\
& 29 \\
& 30 \\
& 31 \\
& 32 \\
& 33 \\
& 34 \\
& 35 \\
& 36 \\
& 37 \\
& 38 \\
& 39 \\
& 40 \\
& 41 \\
& 42 \\
& 43 \\
& 44 \\
& 45 \\
& 46 \\
& 47 \\
& 48 \\
& 49 \\
& 50 \\
& 51 \\
& 52 \\
& 53 \\
& 54 \\
& 55 \\
& 56 \\
& 57 \\
& 58 \\
& 59
\end{aligned}
$$

Figure 2b: Overall survival according to the response to induction chemotherapy

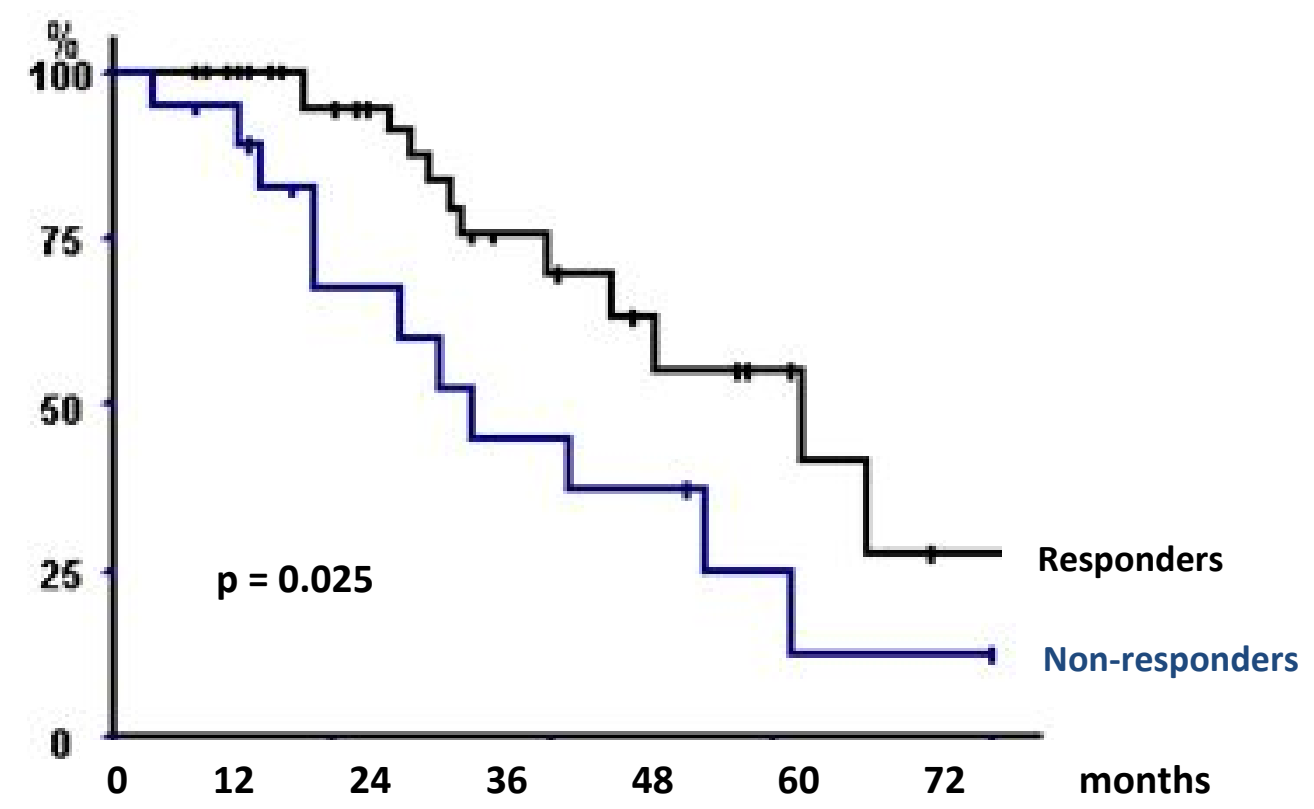

http://mc.manuscriptcentral.com/URO 
Figure 3a: Progression-free survival according to cystectomy achievement

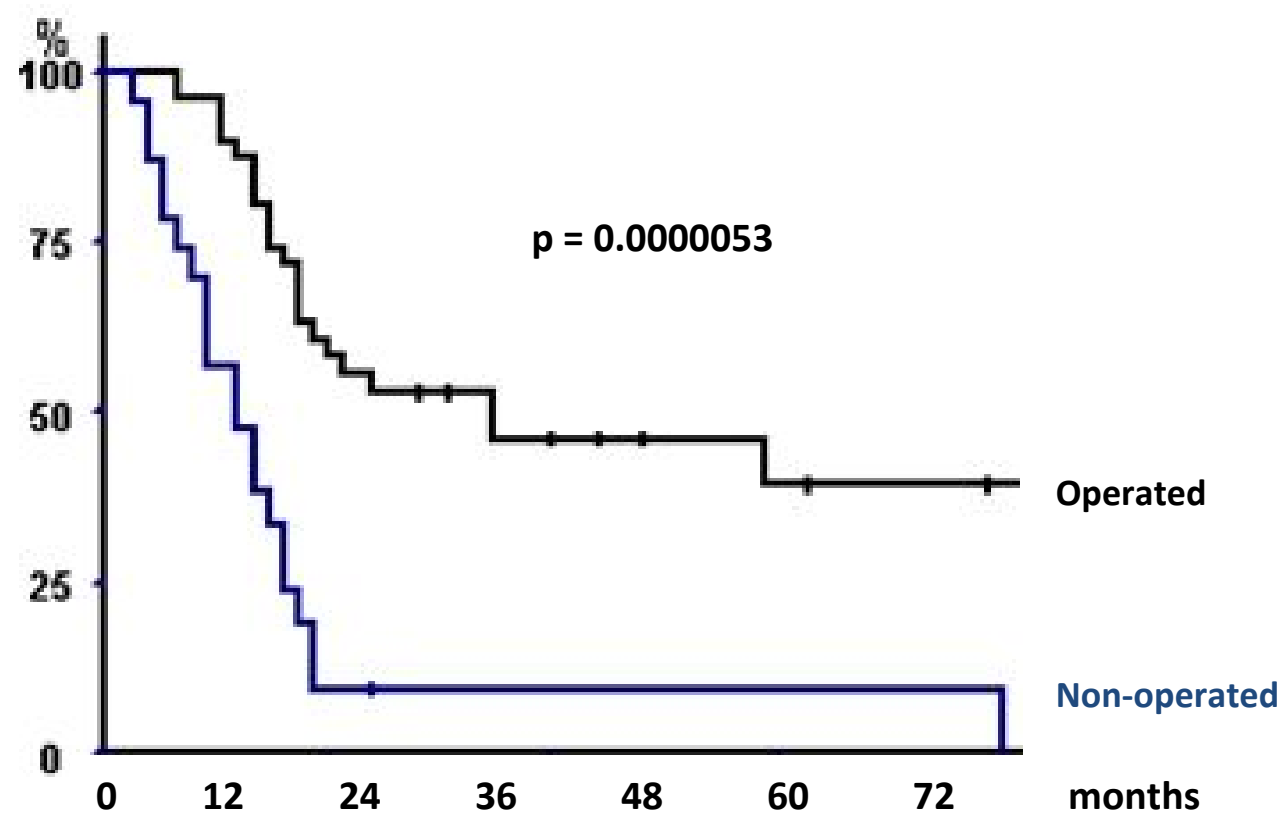


Figure 3b: Overall survival according to cystectomy achievement

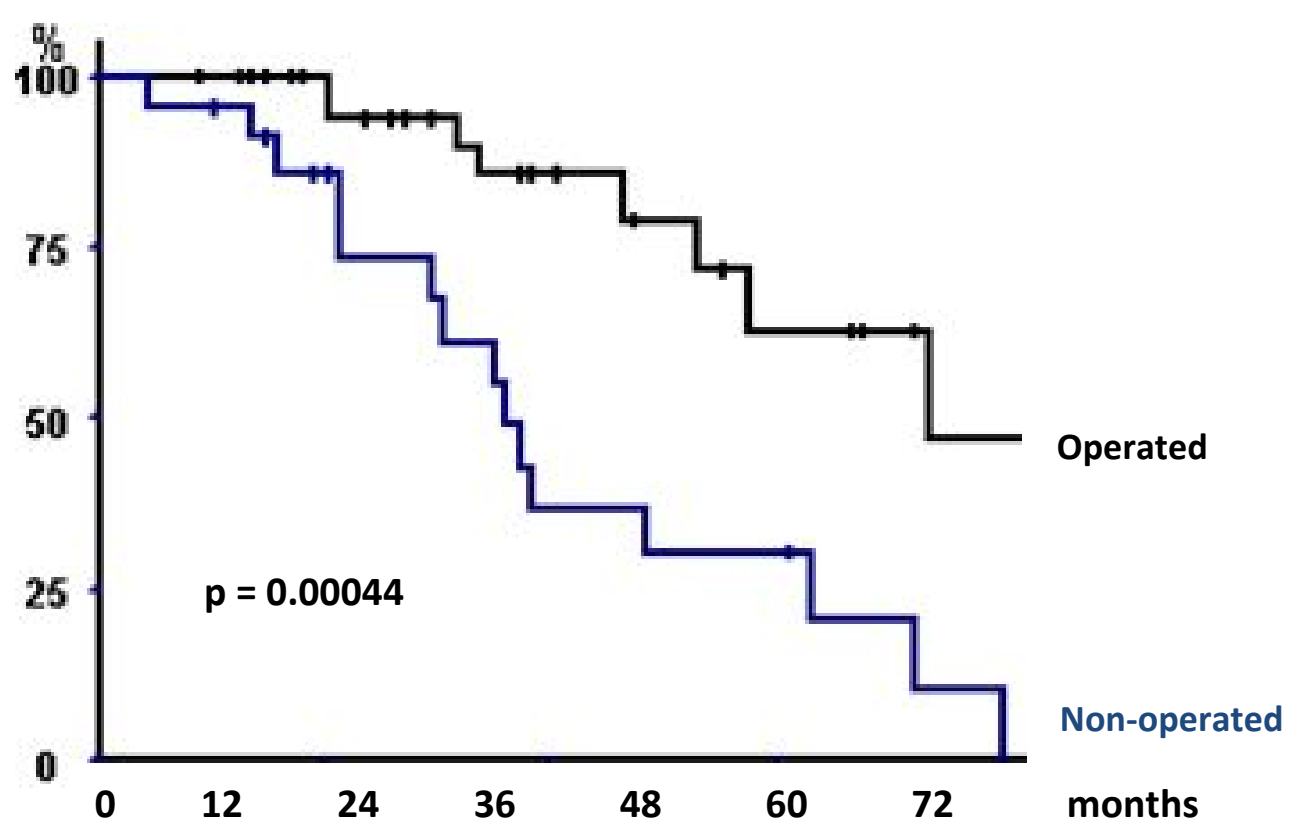

